\title{
Nanogels of a Succinylated Glycol Chitosan-Succinyl Prednisolone Conjugate: Release Behavior, Gastrointestinal Distribution, and Systemic Absorption
}

\author{
Haiyan Zhou, Yuri Ikeuchi-Takahashi, Yoshiyuki Hattori@ and Hiraku Onishi * \\ Department of Drug Delivery Research, Hoshi University, 2-4-41, Ebara, Shinagawa-ku, Tokyo 142-8501, Japan; \\ zhy00630@gmail.com (H.Z.); y-ikeuchi@hoshi.ac.jp (Y.I.-T.); yhattori@hoshi.ac.jp (Y.H.) \\ * Correspondence: onishi@hoshi.ac.jp; Tel.: +81-3-5498-5759; Fax: +81-3-5498-5759
}

Received: 5 March 2020; Accepted: 26 March 2020; Published: 30 March 2020

check for updates

\begin{abstract}
Recently, the potential of nanoparticles (NPs) in ulcerative colitis (UC) therapy has been increasingly demonstrated. Namely, anionic NPs have been found to be accumulated efficiently to the UC damaged area due to epithelial enhanced permeability and retention (eEPR) effect. Previously, a novel anionic nanogel system (NG(S)) was prepared, and evaluated for the efficacy and toxicity. In the present study, release behaviors and biodistribution were investigated in detail to elucidate the functional mechanisms. Rats with 2,4,6-trinitrobenzene sulfonic acid (TNBS)-induced ulcerative colitis (UC) were used as biomodels. In vitro release was examined with or without the contents of the cecum or distal colon. Gastrointestinal distribution and plasma concentrations were investigated after the intragastric administration of $10 \mathrm{mg}$ prednisolone (PD) eq. $/ \mathrm{kg}$. At $\mathrm{pH} 1.2$ and 6.8, release behaviors were slow, but controlled. Overall release was not markedly different irrespective of coexistence of intestinal contents. In in vivo studies, a large amount of PD was distributed in the lower parts of the gastrointestinal tract 6 and $12 \mathrm{~h}$ after administration with NG(S). PD accumulated well in the colonic parts, and prolonged release was noted. The systemic absorption of PD with NG(S) was hardly found. NG(S) concentrated the drug in the colon and showed controlled release. These behaviors were considered to lead to the previously reported good results, promotion of effectiveness and suppression of toxic side effects.
\end{abstract}

Keywords: succinylated glycol chitosan-succinyl prednisolone conjugate; nanogel; gastrointestinal distribution; release behavior; effectiveness; toxic side effect; ulcerative colitis

\section{Introduction}

Although the etiology of ulcerative colitis (UC) remains unclear [1], it is regarded as an autoimmune disease. Additionally, UC is induced by genetic factors or is inherited [2]; however, behavioral and environmental factors also appear to contribute to its onset. UC is a chronic relapsing disease that causes colonic inflammation and complications [3-6]. It is most commonly diagnosed in patients aged between 15 and 35 years, affects $1-2$ million individuals in the United States and 5 million individuals worldwide, and its incidence is increasing in newly industrialized countries [6-8]. Clinically, prednisolone (PD), a typical glucocorticoid, is widely used to treat UC [9-11]. Its current administration methods include oral as well as rectal dosing $[12,13]$. The route of rectal dosing requires maintaining a laying position for a long time, and is not practical for the upper ulcerative colon. When PD is administrated orally, large quantities of the compound are absorbed by the upper digestive tract and may cause severe toxic side effects, including weight gain, diabetes, osteoporosis, and glaucoma [14-16]. Therefore, in the past few years, drug delivery systems (DDSs) have been attracting increasing attention due 
to their potential to promote therapeutic efficacy and reduce severe adverse effects. DDSs using biodegradable polymers [17], $\mathrm{pH}$-dependent controlled release polymers [18,19], enzyme-controlled release polymers [19], gastrointestinal pressure-controlled release [20], and prodrug approaches [21] have been examined and shown to efficiently deliver drugs to inflamed mucosal membranes and suppress systemic absorption [22,23].

In recent decades, the potential of nanoparticles (NPs) in UC therapy has been increasingly demonstrated. The inflamed mucosa of UC is characterized by a deficient intestinal cellular membrane, with the loss of barrier function and increased epithelial permeability. Although these structure accounts for only $1 \%$ of the surface area of the intestinal mucosa, negatively charged NPs may accumulate easily in damaged areas, including epithelial cells and macrophages (Figure 1), because of an increasing concentration of positively charged proteins around there. Such physiological condition is called the epithelial enhanced permeability and retention (eEPR) effect [24,25]. Pathophysiological studies recently revealed that positively charged particles or gels accumulate in normal and healthy membranes, whereas negatively charged ones may localize in the inflamed colonic mucosa [26,27]. Negatively charged NPs are less likely to cohere with mucoadhesion in the upper gastrointestinal tract, and, thus, may be used to target inflamed regions in the colon.

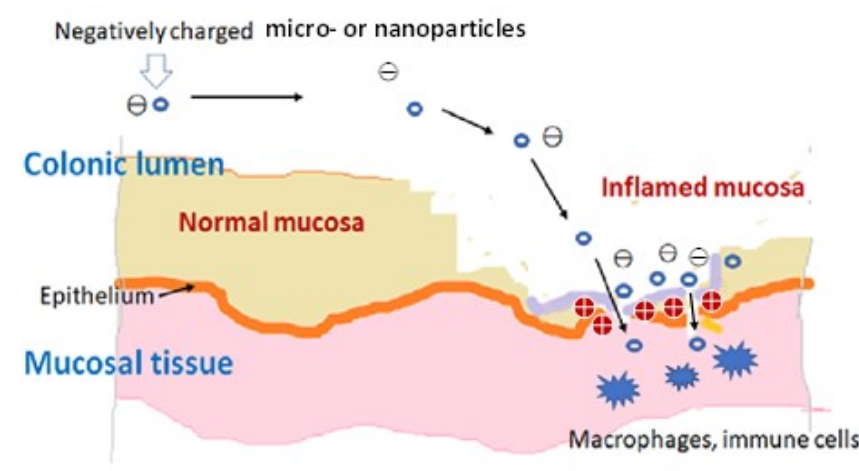

Figure 1. Potential accumulation of nanoparticles (NPs) in intervals of the colonic epithelial cell membrane due to the epithelial enhanced permeability and retention (eEPR) effect.

Previously, we developed the nanogel, NG(S), composed of glycol chitosan-succinyl prednisolone (S-GCh-SP) [26]. NG(S) contains carboxyl esters, and showed gradual release of PD. Such prolonged release by the cleavage of a labile bond such as carboxy esters was attributed to their sensitivities to many elements, including, but not limited to, $\mathrm{pH}$ [26-28].

Based on these findings, the glycol chitosan (GC)- succinyl prednisolone (SP) conjugate, named GCh-SP, was synthesized, and subsequently modified into a negatively charged conjugate by succinic anhydride (SA), named S-GCh-SP. S-GCh-SP with high drug loading forms a nanogel (NG) due to the interactions of hydrophobic PD moieties and functions as a negatively charged NP because of the presence of succinyl groups in the water-soluble polymer parts [28]. In the previous study, the NG of S-GCh-SP, called NG(S), was examined for preparation procedures and particle characteristics, and evaluated mainly for the efficacy and toxicity. In the present study, effect of intestinal contents on release behaviors, gastrointestinal distribution-time profiles and plasma concentrations were investigated in detail to make clear the functional mechanisms.

\section{Results and Discussion}

\subsection{Preparation and Characterization of $N G(S)$}

The structure and PD content of S-GCh-SP could be estimated from the ${ }^{1} \mathrm{H}-\mathrm{NMR}$ data of GCh and S-GCh-SP (supplementary files 1-4). First, as to the structure of original GCh, the substitution degree of glycol groups per chitosan sugar unit was calculated to be $160 \%(\mathrm{~mol} / \mathrm{mol})$, indicating that 
some glycol groups were combined to $\mathrm{C}^{\prime}-\mathrm{OH}$ and other positions, probably $\mathrm{C} 2^{\prime}-\mathrm{OH}$. Additionally, the substitution degree of acetyl groups per chitosan sugar unit was $22 \%(\mathrm{~mol} / \mathrm{mol})$, which was similar to the substitution degree estimated by the supplier. The substitution degree of succinyl groups, calculated from their integrated intensity of $2.2-2.5 \mathrm{ppm}$, was $85 \%(\mathrm{~mol} / \mathrm{mol})$, in which $28 \%(\mathrm{~mol} / \mathrm{mol})$ was derived from the SP part and $55 \%(\mathrm{~mol} / \mathrm{mol})$ was introduced by the reaction using SA. The PD content, estimated from the integrated intensity of typical PD proton, $\mathrm{C} 1-\mathrm{H}$ and that of sugar protons, was $23.4 \%(w / w)$. The PD content, assessed using UV absorption after ester hydrolysis in $0.1 \mathrm{M}$ $\mathrm{NaOH}$ (supplementary file 5), was $20.8 \%$ (w/w; Table 1), and was similar to the content observed from ${ }^{1} \mathrm{H}-\mathrm{NMR}$ data. Generally, the molar number is not necessarily exactly from the integrated intensity in ${ }^{1} \mathrm{H}-\mathrm{NMR}$ because that intensity can be affected by the kinds of structures. Therefore, the PD content was determined by the UV absorbance.

Table 1. Particle characteristics and drug content of NG(S).

\begin{tabular}{cccc}
\hline Preparation & ${\text { Particle Size }(\mathbf{n m})^{\mathbf{1}}}$ & ${\text { Zeta Potential }(\mathbf{m V})^{\mathbf{1}}}$ & ${\text { PD Content }(\mathbf{\%}, \boldsymbol{w} / \boldsymbol{w})^{\mathbf{1}}}^{\mathbf{1}}$ \\
\hline NG(S) & $560 \pm 41$ & $-34.8 \pm 0.3$ & $20.8 \pm 0.4$ \\
\hline \multicolumn{4}{c}{ Results are expressed as the mean \pm S.D. $(n=3)}$.
\end{tabular}

Furthermore, the possibility of SA to react with the hydroxy groups of PD and GCh were checked, which was related to analysis of the release mode. According to the reports by Kato et al. [29], the succinylation proceeded preferentially at the amino groups in the present reaction conditions. In addition, when the reactivity of SA to SP was checked in the similar reaction conditions, no succinylation of SP was caused, indicating the hydroxy groups of SP should not be succinylated by SA. Moreover, in the release study from NG(S) in the aqueous media of $\mathrm{pH} 1.2$ and 6.8, only PD was recognized to be released, and ester forms of PD with SA were hardly detected (see Section 2.2. In vitro Release from NG(S)). These supported that only the simple $N$-succinylation of GCh-SP by SA should be caused.

The particle characteristics of NG(S) are shown in Table 1. The mean size was $560 \mathrm{~nm}$ and mean zeta potential was $-34.8 \mathrm{mV}$. Its size distribution is shown in Figure 2. As to the size distribution of $\mathrm{NG}(\mathrm{S})$, the polydiversity index, shown as a value of (size distribution width represented by standard deviation)/(mean size in the distribution) was approximately 0.28 . The PD content was $20.8 \%(w / w)$, which was larger than the previous one. The reaction conditions, particularly, $\mathrm{pH}$ adjustments in GCh-SP conjugation and reaction with SA appeared to influence the PD content.

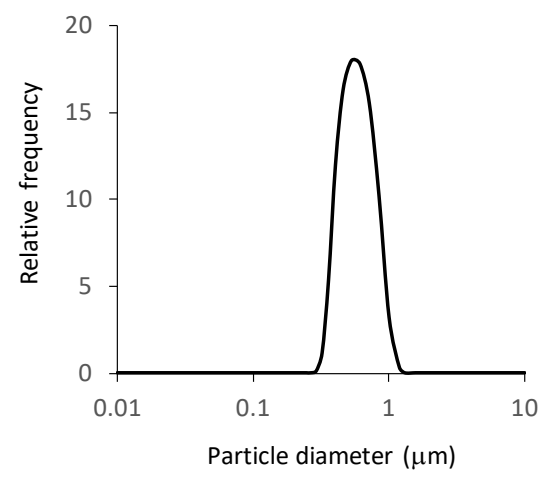

Figure 2. Particle size distribution of NG(S).

Although structure analyses by other measurements were not performed, spectra data such as MS or FT-IR measurement will enable the structural analysis in the form of powder. These data might be needed in the following study. 


\subsection{In Vitro Release from $N G(S)$}

NG(S) samples with and without cecal or distal colonic contents were incubated, and aliquot samples were collected at appropriate time points and analyzed by high-performance liquid chromatography (HPLC). Release profiles were obtained from free PD detected (detection limit $=0.01 \mathrm{mg} / \mathrm{mL}$ ). Based on a previously reported validation study, free PD evaluated by the direct injection in HPLC reflected that from NG(S) to the outer medium; the free PD concentration obtained using the ultrafiltration membrane (MW cut-off with 30,000) was the same as that of the present method. Therefore, release experiments were conducted in a similar manner.

In the present study, drug release profiles from NG(S) were investigated in JP 17 first fluid ( $\mathrm{pH}$ 1.2), JP 17 s fluid ( $\mathrm{pH} 6.8$ ), and a cecal or colonic content suspension $(20 \%, w / v)$ in JP 17 second fluid. The first and second fluids are commonly used as simulated gastric and small intestinal media, respectively, and the difference in $\mathrm{pH}$ should influence the hydrolysis rate of the ester of the SP moieties in NG(S). Although the medium $\mathrm{pH}$ around the diseased area was not known exactly, that was presumed to be weakly acidic to neutral. In this study, the second fluid was used. Release profiles were obtained as shown in Figure 3. Under conditions with no intestinal contents, release was very slow, mean cumulative release ratios gradually increased to approximately $1 \%$ and $2 \%(w / w)$ at 24 and $48 \mathrm{~h}$, respectively, and the difference in the release rate between $\mathrm{pH} 1.2$ and 6.8 was small.

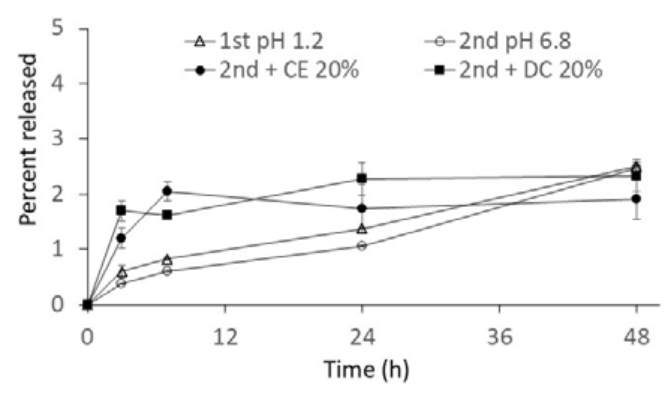

Figure 3. Release profiles from NG(S) with a high drug content (21\%) in media of JP 17 first fluid (1st $\mathrm{pH} 1.2$ ) and JP $17 \mathrm{~s}$ fluid (2nd pH 6.8) without or with a $20 \%$ cecal content homogenate (2nd + CE 20\%) and $20 \%$ distal colonic content homogenate ( 2 nd + CD 20\%). Results are expressed as the mean \pm S.D. $(n=3)$.

As more SP moieties promote hydrophobic interaction in NG(S), its physical state would be changed. Considering the drug contents, the hydrophobic interaction might increase in the present NG(S) as compared with the previous one (less PD content). On the other hand, the carboxylates of succinyl moieties should cause the repulsion electrically each other and influence the microenvironment of the ester bond. Such change in the physical state might affect the release rate in the present NG(S); more carboxylates around the ester bond might bring about more acidic microenvironment, leading to less hydrolysis rate of the ester.

Moreover, the release rate was affected by the addition of intestinal contents. The initial release rate initially accelerated; however, the effect was small. The slight increase observed in the amount released may have been due to some enzymes already present in the homogenate. However, the macromolecular structures and hydrophobic association of SP moieties give steric hindrance to the enzymes. Therefore, the influence of intestinal contents on release was considered to have been small. Effect of intestinal contents on the hydrolysis of ester prodrugs had been reported before [30-32], and intestinal fluid of the cecal and colonic regions appeared to act as the degradation of the ester prodrugs. Although the high molecular weight ester prodrug is not easily degraded by the esterase alone, the ester hydrolysis can be promoted after the degradation of the backbone. Udo et al. reported that the high molecular weight ester could be promoted by the addition of rat cecal contents, which was because the cecal content contained the esterase and enzymes related to the breakdown of sugar backbones [32]. The release of 
PD from NG(S) was accelerated, though the release extent was small. The initial rapid release might be due to the enzymatic effect by the intestinal contents.

In our previous study, the release rate was dependent on the medium $\mathrm{pH}$. Hydrophilic macromolecules in NG(S) were swollen by immersion in aqueous media, and PD, liberated by ester hydrolysis, was released into the media. In the present study, pH-dependent release was not marked. Furthermore, the release rate was slower than that reported previously [26]. The present NG(S) had a higher content of PD $(20.8 \%(w / w))$ than the previous study $(13.7 \%(w / w))$ [26]. In NG(S) with a high drug content, the hydrophobic interaction of the SP moieties was considered to be strong. In these rigid structures, the ester of the SP moieties may not have been subjected to hydrolysis, leading to the smaller release of free PD. Similar features were reported for the NG with a higher PD content; the chondroitin sulfate-glycyl prednisolone conjugate nanogel (CS-GP-NG), in which CS-GP-NG with a higher PD content showed a smaller release rate and had a weaker influence on the difference caused by medium $\mathrm{pH}$ [33]. The difference in PD content of NG(S) appeared to influence the release rate and stability of the ester bond.

In the cecal or colonic content suspension $(20 \%, v / v)$ in JP $17 \mathrm{~s}$ fluid, only the initial release rate was slightly increased, and the later release became slow. Various components contained in the intestinal contents may interact with the surface molecules of NG(S). Such interaction of the medium component with NPs was reported by Zhang et al. [34]. The modification of NG(S) by the components of the intestinal contents might influence the release patterns. Similar phenomena were reported for chitosan-SP conjugate microspheres [35], the release rate of which in a $20 \%(w / v)$ intestinal suspension had been suppressed to nearly $50 \%$ of that in the buffer alone.

Finally, the released compounds were confirmed in order to assure that only PD was released in the media. It was demonstrated in the following experimental results. (1) SP did not react with SA in a similar reaction condition. (2) The PD concentration in the ultrafiltrate was not changed after the further $24 \mathrm{~h}$ incubation of the ultrafiltrate. (3) After the ultrafiltrate was treated in $0.1 \mathrm{M} \mathrm{NaOH}$, the PD concentration did not increase. These results supported that only PD was released and other PD esters were not existed.

\subsection{Drug Distribution in the Gastrointestinal Tract after the Intragastric Administration of NG(S)}

The distribution of the drug in the gastrointestinal tract of UC rats was investigated after the intragastric administration of $\mathrm{NG}(\mathrm{S})$ and compared with that after dosing with PD alone. The gastrointestinal tract of 2,4,6-trinitrobenzene sulfonic acid (TNBS)-induced UC rats was divided into 6 parts: the stomach (ST), proximal small intestine (PI), distal small intestine (DI), cecum (CE), proximal colon (PC), and distal colon (DC), on which the operation was performed such that the length of PI $=$ the length of DI and the length of DC $=2 \times$ the length of PC. The contents of each part were removed with a spatula, including mucosal surface materials. Free and total (free + conjugated) PD were investigated, and the distribution profiles for NG(S) were shown in Figure 4. Free and total drugs were both observed mainly in the stomach and small intestine at $3 \mathrm{~h}$. Most of PD was detected as the conjugated form, the amount of which was 10-fold or higher than that of free PD. Six and twelve hours after dosing, a large amount of the drug was distributed in the CE, PC, and DC. In these periods, the amount of conjugated PD was 10-fold or higher than that of free PD. These results indicated that $\mathrm{NG}(\mathrm{S})$ transfers well to the large intestinal parts containing the colon, and that free PD may be slowly released there. Furthermore, NG(S) distributed in the large intestine appeared to remain there for a long period. These drug distribution patterns suggested that NG(S) efficiently targeted the colonic diseased part. NG(S) distributed in the inflamed area gradually released PD, which may be taken up by inflammatory cells, leading to the suppression of inflammation. The amount of total PD recovered in the gastrointestinal tract was nearly $20 \%(w / w)$ at $3 \mathrm{~h}$, and approximately $15-16 \%(w / w)$ at 6 and $12 \mathrm{~h}$. Low recovery ratios were considered to be attributed to (1) the contents not being completely collected with a spatula, in particular, NG(S), attached to the mucosal surface, potentially remaining there, and (2) the decomposition of PD itself in the intestinal fluids; though it was not checked in 
detail. Since recovery ratios were similar between 6 and $12 \mathrm{~h}, \mathrm{NG}(\mathrm{S})$ appeared to be fairly stable in the gastrointestinal tract.

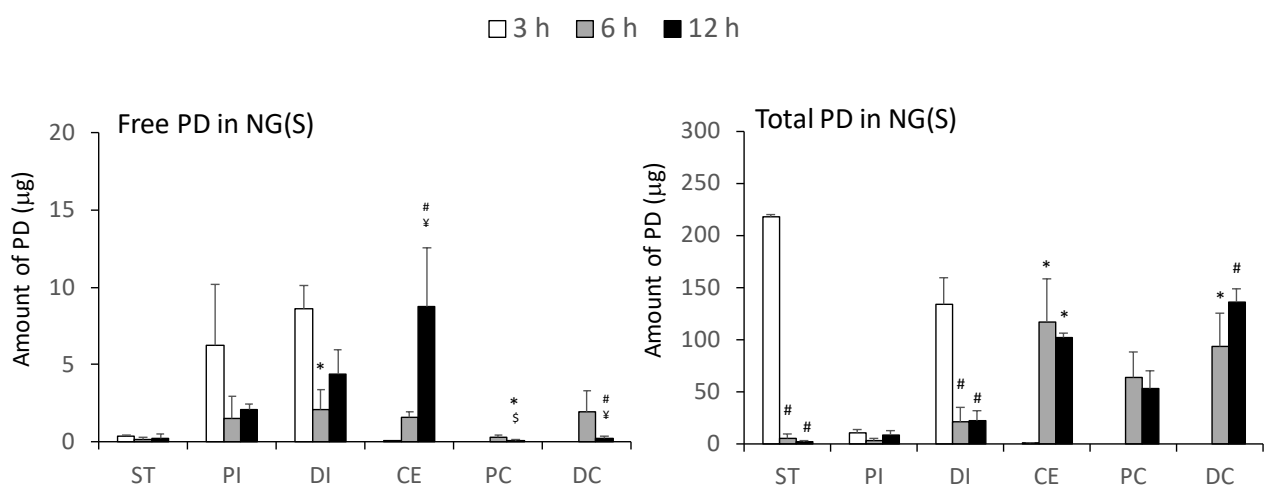

Figure 4. Gastrointestinal distribution of PD after the oral administration of NG(S) and PD alone to rats with TNBS-induced UC at $10 \mathrm{mg}$ PD eq. $/ \mathrm{kg}$. Results are expressed as the mean \pm S.E. ( $n=4$, except for the stomach, $1 \mathrm{~h}(n=3))$. ${ }^{*} p<0.05$ vs. $3 \mathrm{~h} ;{ }^{\#} p<0.01$ vs. $3 \mathrm{~h} ;{ }^{\$} p<0.05$ vs. $6 \mathrm{~h}:{ }^{*} p<0.01$ vs. $6 \mathrm{~h}$.

Drug distribution after the dosing of PD alone at $10 \mathrm{mg} / \mathrm{kg}$ in a similar UC model has already been reported by Yano et al. [14]; the whole tissue (contents + tissue) was homogenized and the homogenate was used in a distribution study. Drug distribution following the administration of PD alone appeared to be limited to the upper gastrointestinal tract. After $3 \mathrm{~h}$, the majority of PD was recovered in the stomach and small intestinal parts, and the total amount recovered was approximately $20 \%$. Furthermore, PD was not detected 6 or $12 \mathrm{~h}$ after dosing. PD orally administered at $5 \mathrm{mg} / \mathrm{kg}$ was previously shown to be absorbed well and eliminated quickly from the systemic circulation [35]. Therefore, NG(S) was considered to move more gradually and efficiently to the lower intestine than PD alone, resulting in enhancement of delivery to the diseased area.

\subsection{Plasma Concentration of PD after the Intragastric Administration of NG(S)}

Dissolved PD is absorbed well in the upper intestine [35-37]. Furthermore, according to the study by Yano et al. [14], PD was quickly absorbed into the systemic circulation after oral dosing with a suspension of PD alone at $10 \mathrm{mg} / \mathrm{kg}$. This good absorption of free PD is associated with side effects $[14,34]$. Therefore, the plasma PD levels detected were considered to be an indicator of the severity of side effects. In the present study, plasma samples were collected immediately before each excision time point $(3,6$, and $12 \mathrm{~h})$ in gastrointestinal distribution studies (Figure 5). A mean plasma level of free PD was detected at approximately $0.01 \mu \mathrm{g} / \mathrm{mL} 3 \mathrm{~h}$ after dosing. The plasma level of free PD was below the detection limit $(0.01 \mu \mathrm{g} / \mathrm{mL})$ at 6 and $12 \mathrm{~h}$. The negligible appearance of PD in the plasma after the intragastric dosing of NG(S) suggested a decrease in side effects with NG(S).

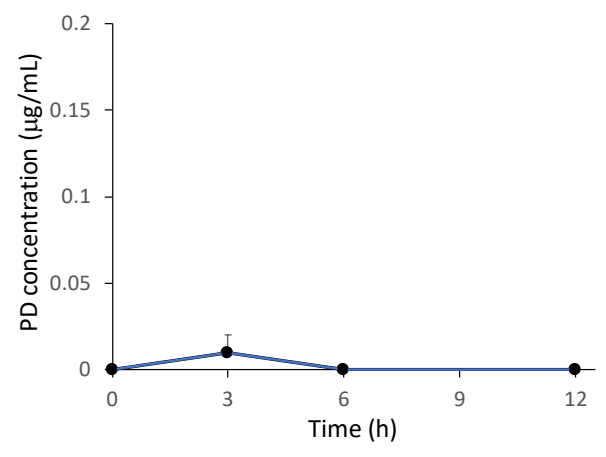

Figure 5. Time course of plasma PD levels after dosing of NG(S). Each point represents the mean \pm S.E. of four rats. 


\section{Materials and Methods}

\subsection{Materials and Animals}

\subsubsection{Materials}

Glycol chitosan (GCh; MW = ca. 100,000, deacetylation degree = approximately 80\% $(\mathrm{mol} / \mathrm{mol})$ ), PD, SP, SA, and 1-ethyl-3-(3-dimethylaminopropyl) carbodiimide hydrochloride (WSC) were purchased from Wako Pure Chemical Industries, Ltd. (Osaka, Japan). TNBS was purchased from Sigma Chemical Company (St. Louis, MO, USA). Sephadex G50 (fine grade) was obtained from GE Healthcare Bio-Sciences AB (Uppsala, Sweden). Pentobarbital solution, named Somnopentyl, was purchased from Kyoritsu Seiyaku Corporation (Production No. 7231101, Tokyo, Japan). All other chemicals were of reagent grade.

\subsubsection{Animals}

Male Wistar rats (7 weeks old, 200-210 g) were purchased from Tokyo Laboratory Animal Science Co., Ltd. and raised in a room with a 12-h light-dark cycle. Room temperature and relative humidity were maintained at $23 \pm 1{ }^{\circ} \mathrm{C}$ and $60 \% \pm 1 \%$, respectively. Animals were fed with the specific breeding diet MF provided by Oriental Yeast Co., Ltd. (Tokyo, Japan) with water available ad libitum. The experimental protocol was approved by the committee on Animal Research of Hoshi University (Tokyo, Japan; project identification code: 26-048, approval date: 20 April 2018) and animal experiments were performed in accordance with the Guiding Principles for the Care and Use of Laboratory Animals of Hoshi University.

\subsection{Equipment}

UV-VIS absorption was measured with a Beckman DU640 spectrophotometer (Beckman Coulter, Inc., Brea, CA, USA). HPLC was used to detect PD with the Shimadzu SPD-10AV VP UV-VIS detector set at a wavelength of $246 \mathrm{~nm}$ and Shimadzu C-R7A plus Chromatopac (Kyoto, Japan). The size and $\zeta$-potentials of the particles produced were measured using an ELS-Z2 apparatus (Otsuka Electronic Co., Ltd., Osaka, Japan). ${ }^{1}$ H-NMR spectra were measured with a JNM-ECA600 II spectrometer (JEOL, Tokyo, Japan).

\subsection{Preparation of GCh-SP and S-GCh-SP}

The preparation scheme of GCh-SP and S-GCh-SP is shown in Figure 6A. These conjugates were produced in a similar manner to that described previously [28]. During the reaction, $1 \mathrm{M} \mathrm{HCl}$ and $1 \mathrm{M}$ $\mathrm{NaOH}$ were used to adjust $\mathrm{pH}$. GCh $(100 \mathrm{mg})$ was dissolved in $15 \mathrm{~mL}$ water, $\mathrm{pH}$ was adjusted to 6.2-6.3, and SP (100 mg) was dissolved in $5 \mathrm{~mL}$ of tetrahydrofuran (THF). After mixing evenly, the pH of the resulting material was adjusted to approximately 6.3. WSC (500 mg) was gradually added and stirred under ice bath conditions for $1 \mathrm{~h}$, and the reaction was then continued at room temperature $\left(25^{\circ} \mathrm{C}\right)$ for another $23 \mathrm{~h}$. After gel chromatography, macromolecular fractions were collected and dialyzed extensively against water using a cellulose tube. The residue in the tube was obtained as an aqueous suspension of the NG of GCh-SP, named NG(G). In the next step, THF and 0.5 M phosphate buffer ( $\mathrm{pH}$ 7) were added to aqueous medium containing $\mathrm{NG}(\mathrm{G})(150 \mathrm{mg})$ at a ratio of $1 / 3(v / v)$ and an excessive amount of SA (1050 mg) was then gradually added and stirred for $3 \mathrm{~h}$. Throughout this period, $\mathrm{pH}$ and temperature were maintained at approximately 7.0 and $15^{\circ} \mathrm{C}$, respectively. The mixture was subjected to gel chromatography, and high-molecular-weight fractions were collected and dialyzed in a similar method to that for $\mathrm{NG}(\mathrm{G})$. The resultant mixture was obtained as an aqueous suspension of a NG of S-GCh-GP, named NG(S) (Figure 6B). 
A

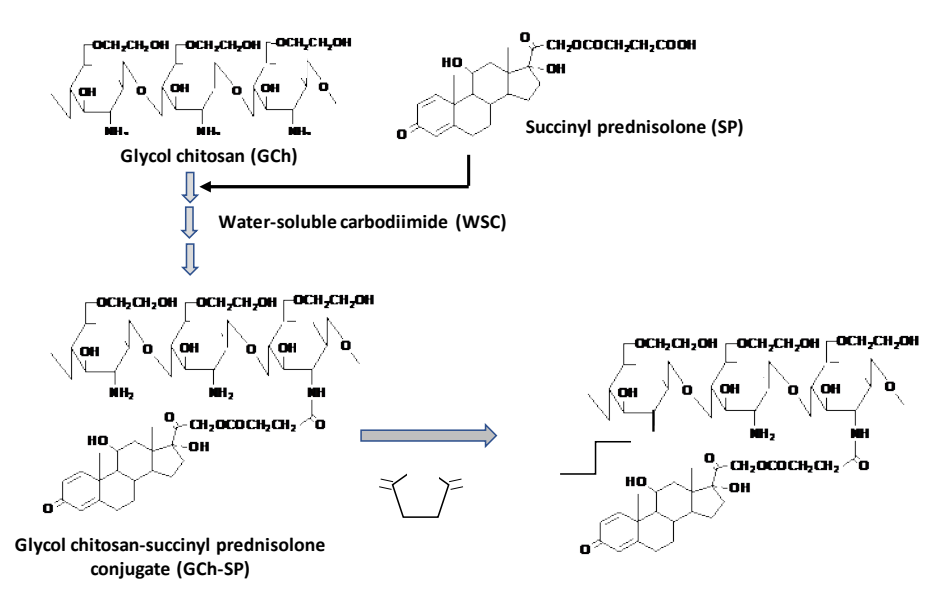

B

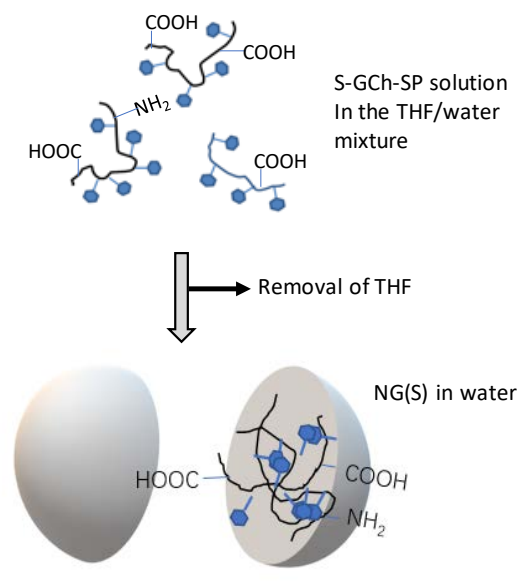

Figure 6. (A) Synthetic scheme of GCh-SP and S-GCh-SP. GCh: glycol-chitosan, SP: succinyl prednisolone, GCh-SP: GCh-SP conjugate, S-GCh-SP: succinylated GCh-SP, WSC: water-soluble carbodiimide, SA: succinic anhydride. (B) A diagram showing the formation of NG(S) in water.

The structure of S-GCh-SP was confirmed from its ${ }^{1} \mathrm{H}-\mathrm{NMR}$ spectrum. The mixture of dimethylsulfoxide (DMSO)- $\mathrm{d}_{6} / \mathrm{D}_{2} \mathrm{O}(1: 1, v / v)$ was used as a solvent. Tetramethylsilane (TMS) was used as a standard $(0 \mathrm{ppm})$ for the proton signal. The PD content of each NG was investigated as follows: $\mathrm{NG}(\mathrm{G})$ and $\mathrm{NG}(\mathrm{S})$ were heated at $45^{\circ} \mathrm{C}$ for $10 \mathrm{~min}$ in $0.1 \mathrm{M} \mathrm{NaOH}$ aqueous solution. The solutions were then centrifuged. The supernatants were collected and spectrophotometrically measured at $246 \mathrm{~nm}$. The PD content was calculated based on the absorbance of free PD in $0.1 \mathrm{M} \mathrm{NaOH}$ aqueous solution.

\subsection{Production of the UC Model}

UC animal models were produced by treating rats with TNBS $[23,38,39]$. TNBS was dissolved in $50 \%(v / v)$ ethanol aqueous solution at a concentration of $80 \mathrm{mg} / \mathrm{mL}$. After rats were fasted for 2 days, TNBS solution $(0.25 \mathrm{~mL})$ was instilled into the colon of each rat, $8 \mathrm{~cm}$ from the anus, using a catheter.

\subsection{In vitro Release Studies}

In vitro release experiments were performed using JP 17 first fluid ( $\mathrm{pH}$ 1.2), JP $17 \mathrm{~s}$ fluid ( $\mathrm{pH}$ 6.8), and JP 17 second fluid containing $20 \%(w / v)$ cecal or $20 \%(w / v)$ distal colonic contents, in which the distal colon (DC) meant the latter 2/3 part of the whole colon. The NG(S) suspension in JP 17 first or second fluid with no intestinal contents was prepared at a concentration of $40 \mu \mathrm{g}$ PD eq./mL. Regarding release tests with intestinal contents, incubation samples were prepared as follows. Three days after the instillation of TNBS, rats were sacrificed by injecting excessive Somnopentyl intraperitoneally, and the wet cecum (CE) or DC was collected, weighed, and diluted at 20\% using JP 17 second fluid. The diluted contents were further homogenized with a glass homogenizer using a Teflon pestle, and homogenates were filtered with gauze and used to prepare incubation samples. The final incubation sample was set as NG(S) in a cecal or distal colonic suspension $(20 \%(w / v)$ wet content) at a concentration of $50 \mu \mathrm{g}$ PD eq./mL.

Each mixture was incubated by horizontal shaking at $60 \mathrm{rpm}$ at $37^{\circ} \mathrm{C}$. At appropriate time points, a small portion of the suspension was withdrawn from each mixture and processed by the method described below.

Regarding the mixture with no intestinal contents, immediately after each cycle of sampling, $100 \mu \mathrm{L}$ of $0.1 \mathrm{M}$ acetate buffer $(\mathrm{pH} 4)$ was mixed with each sample taken $(50 \mu \mathrm{L})$ to suppress further release. The resultant mixture was analyzed by HPLC to assess the release of PD. Regarding the incubation with intestinal contents, after each cycle of sampling, $100 \mu \mathrm{L}$ of water was mixed with each 
sample taken $(100 \mu \mathrm{L})$ using a vortex mixer. Methanol $(140 \mu \mathrm{L})$ and $20 \%(w / v)$ trichloroacetic acid aqueous solution $(40 \mu \mathrm{L})$ were added separately. The mixture was stirred vigorously with a vortex mixer. After centrifugation at $3000 \mathrm{rpm}$ for $10 \mathrm{~min}$, the supernatant was analyzed by HPLC.

\subsection{Drug Distribution Studies in the Gastrointestinal Tract after Intragastric Administration}

TNBS-induced UC model rats were prepared as described above. Three days after the instillation of TNBS, twelve rats were divided into three groups $(n=4)$, and fed with water available ad libitum, but fasted for $24 \mathrm{~h}$ prior to intragastric administration. Each rat was administrated $1 \mathrm{~mL}$ of the $\mathrm{NG}(\mathrm{S})$ suspension (equivalent to $10 \mathrm{mg} \mathrm{PD} / \mathrm{kg}$ ). At appropriate intervals $(3,6$, and $12 \mathrm{~h}$ ), rats were sacrificed by injecting excessive Somnopentyl intraperitoneally (Figure 7A). The gastrointestinal tract was cut and divided into six parts: ST, PI (anterior half of the small intestine), DI (posterior half of the small intestine), CE, PC (1/3 front side of the colon), and DC (2/3 rear side of the colon; Figure 7B). The contents in each part were collected by washing with cold PBS (pH 7.4). The contents of ST, CE, and DC were homogenized with a glass homogenizer with a Teflon pestle under ice bath conditions, and diluted to $8 \mathrm{~mL}$ with cold PBS ( $\mathrm{pH}$ 7.4), while the other contents were similarly homogenized, but diluted to $4 \mathrm{~mL}$ using cold PBS (pH 7.4).

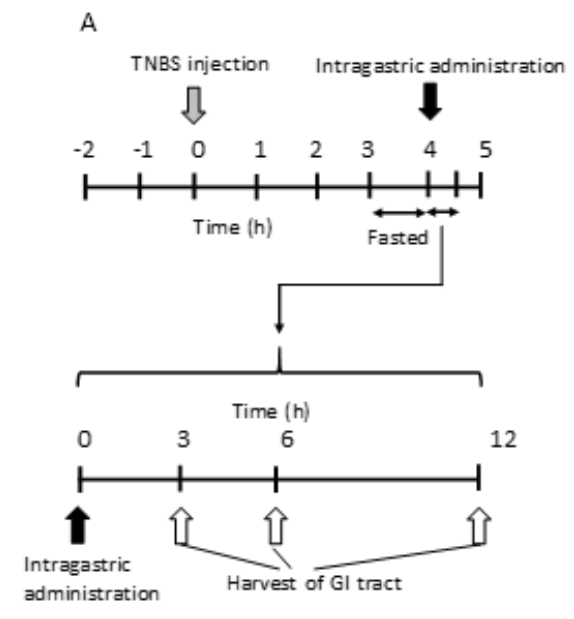

B

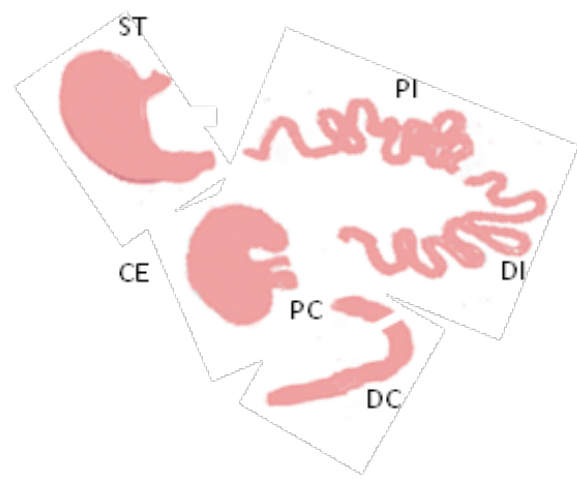

Figure 7. Animal experiment procedure of drug distribution in the gastrointestinal tract of UC model rats. (A) After fasting for $48 \mathrm{~h}$, TNBS was injected from the anus, and 3 days later, rats that lost $20 \%$ of their body weight were fasted for another $24 \mathrm{~h}$, subjected to intragastric dosing, and sacrificed after 3 , 6, and $12 \mathrm{~h}$. (B) Diagram of the division of the gastrointestinal tract. ST, stomach; PI, proximal small intestine; DI, distal small intestine; and CE, cecum; PC, proximal colon; DC, DC. The length of PI = the length of DI; The length of DC $=2 \times$ the length of PC.

Free and total (free + conjugated) PD concentrations in the samples collected were assessed as follows. (1) Processing to detect free PD: this was performed in a similar manner to in vitro studies. Water $(100 \mu \mathrm{L})$ was mixed with each sample collected $(100 \mu \mathrm{L})$ using a vortex mixer. Methanol $(140 \mu \mathrm{L})$ and $20 \%(w / v)$ trichloroacetic acid aqueous solution $(40 \mu \mathrm{L})$ were added separately. The mixture was then stirred vigorously with a vortex mixer. After centrifugation for $10 \mathrm{~min}$ at $3000 \mathrm{rpm}$, the supernatant was analyzed by HPLC. (2) Processing to detect total PD: $50 \mu \mathrm{L}$ of $0.1 \mathrm{M} \mathrm{NaOH}$ was added to $50 \mu \mathrm{L}$ of the homogenate, followed by an incubation at $45^{\circ} \mathrm{C}$ for $10 \mathrm{~min}$. After that, $50 \mu \mathrm{L}$ of $0.1 \mathrm{M} \mathrm{HCl}$ and $50 \mu \mathrm{L}$ of water were added to the mixture to neutralize alkali, then stirred vigorously with a vortex mixer. Methanol $(140 \mu \mathrm{L})$ and $20 \%(w / v)$ trichloroacetic acid aqueous solution $(40 \mu \mathrm{L})$ were added separately. The mixture was stirred vigorously with a vortex mixer. After centrifugation at $3000 \mathrm{rpm}$ for $10 \mathrm{~min}$, the supernatant was analyzed by HPLC. 


\subsection{HPLC Assay}

HPLC was performed at room temperature $\left(25^{\circ} \mathrm{C}\right)$ using a Shimadzu LC-6AD pump with a Shimadzu SPD-10AV VP UV-VIS detector set at a wavelength of $246 \mathrm{~nm}$ and Shimadzu C-R7A plus Chromatopac (Kyoto, Japan). A YMC Pack ODS-AM column (I.D. of $6 \mathrm{~mm}$, length of $150 \mathrm{~mm}$; YMC Co., Ltd., Kyoto, Japan) was used as the analytical column. A mixture of acetonitrile and $50 \mathrm{mM}$ citrate buffer with $\mathrm{pH}$ adjusted to 4.1 with phosphoric acid $(35: 65, v / v)$ was used as the mobile phase. The injection volume was $20 \mu \mathrm{L}$, and the flow rate was set at $1 \mathrm{~mL} / \mathrm{min}$. PD concentrations were assessed using an absolute calibration curve.

\subsection{Statistical Analysis}

A one-way analysis of variance (ANOVA), followed by Scheffé's post hoc test, was used to assess the significance of differences. A significant difference was set as $p<0.05$.

\section{Conclusions}

In the traditional method used to treat severe UC, a typical steroid anti-inflammatory drug, PD is highly effective in clinical practice; however, the long-term use of PD is associated with severely toxic side effects mainly due to its non-specific body distribution, resulting in various hormonal actions. Researchers in various countries are attempting to overcome these issues by suppressing non-specific drug distribution and promoting efficient drug delivery to the target site, leading to the decrease in the amount of drug administered. In recent decades, NPs have been attracting interest in UC therapy. Due to their pathophysiological characteristics, negatively charged NPs specifically accumulate at the diseased site of UC and are phagocytosed by inflammatory cells around these areas. Such NPs, accumulating in damaged membrane and inflammatory cells, can stay long in the damaged region.

In the present study, a negatively charged NG, NG(S), was successfully prepared and its targeted drug delivery profiles, gastrointestinal distribution, plasma concentration, and release behavior were evaluated. The distribution results of NG(S) showed that it moved smoothly to the lower gastrointestinal parts, and remained well in the lower intestine including colon, as compared with PD alone. Plasma concentration profiles showed that PD was hardly absorbed into the systemic circulation after dosing with NG(S). Moreover, NG(S) with a higher PD content $(20.8 \%)$ exhibited very slow release under acidic and neutral environments. The slower release by the present NG(S) with a PD content of $20.8 \%$ might be attributed to its more compact structures formed by the hydrophobic interaction among succinyl prednisolone moieties, resulting in the suppression of accessibility of water-based media. These release characteristics and biodistribution features of NG(S) make it suitable as a DDS for UC therapy.

Supplementary Materials: The following are available online at http://www.mdpi.com/1422-0067/21/7/2376/s1.

Author Contributions: Conceptualization, H.O.; methodology, H.O. and H.Z.; investigation, H.O., H.Z., Y.I.-T. and Y.H.; writing-original draft preparation, H.Z.; writing-review and editing, H.O.; project administration, H.O. All authors have read and agreed to the published version of the manuscript.

Funding: This research received no external funding.

Conflicts of Interest: The authors declare no conflicts of interest.

\section{Abbreviations}

$\begin{array}{ll}\text { CE } & \text { cecum } \\ \text { DMSO } & \text { dimethyl sulfoxide } \\ \text { DC } & \text { distal colon } \\ \text { DI } & \text { distal small intestine } \\ \text { e-EPR } & \text { epithelial enhanced permeability and retention } \\ \text { GCh } & \text { glycol chitosan }\end{array}$




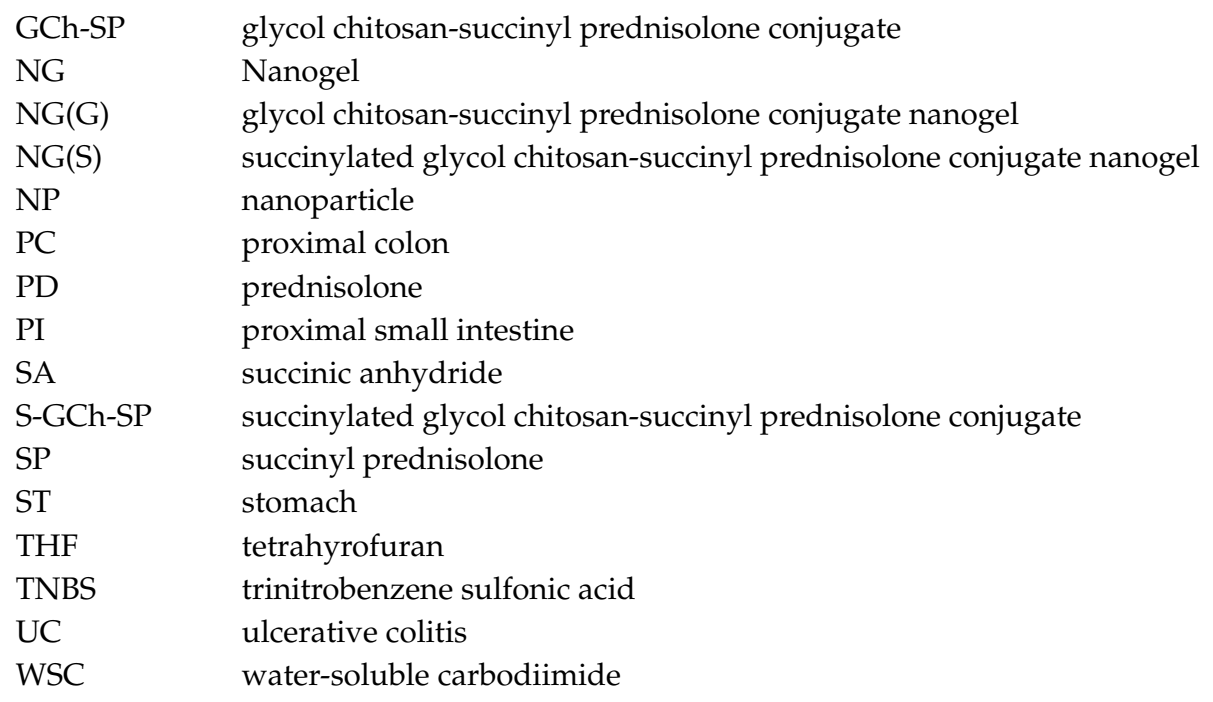

\section{References}

1. Gallo, G.; Kotze, P.G.; Spinelli, A. Surgery in ulcerative colitis: When? How? Best Pract. Res. Clin. Gastroenterol. 2018, 32-33, 71-78. [CrossRef] [PubMed]

2. Medrano, L.M.; Pascual, V.; Bodas, A.; López-Palacios, N.; Salazar, I.; Espino-Paisán, L.; González-Pérez, B.; Urcelay, E.; Mendoza, J.L.; Núñez, C. Expression patterns common and unique to ulcerative colitis and celiac disease. Ann. Hum. Genet. 2019, 83, 86-94. [CrossRef] [PubMed]

3. Lakatos, P.L.; Lakatos, L. Risk for colorectal cancer in ulcerative colitis: Changes, causes and management strategies. World J. Gastroenterol. 2008, 14, 3937-3947. [CrossRef]

4. Nakata, Y.; Nakashima, Y.; Kubota, N.; Kaiga, T.; Mamiya, T.; Mihara, Y.; Yamasaki, Y.; Jinno, D.; Kobayashi, M.; Miyata, T.; et al. A case of fulminant ulcerative colitis complicated with toxic megacolon and perforation. J. Nihon Univ. Med. Assoc. 2013, 72, 26-29.

5. Kirk, A.P.; Cason, J.; Fordham, J.N.; Brown, K.A.; Goddard, D.H.; Holborow, E.J.; Lennard-Jones, J.E. Polymorphonuclear leukocyte function in ulcerative colitis and Crohn's disease. Dig. Dis. Sci. 1983, 28, 236-248. [CrossRef]

6. Nunes, R.; Neves, J.D.; Sarmento, B. Nanoparticles for the regulation of intestinal inflammation: Opportunities and challenges. Nanomedicine 2019, 14, 2631-2644. [CrossRef]

7. Kane, S.V. Systematic review: Adherence issues in the treatment of ulcerative colitis. Aliment. Pharmacol. Ther. 2006, 23, 577-585. [CrossRef]

8. Head, K.A.; Jurenka, J.S. Inflammatory bowel disease part I: Ulcerative colitis-pathophysiology and conventional and alternative treatment options. Altern. Med. Rev. 2003, 8, 247-283.

9. Ishii, N.; Chiba, M.; Suzuki, T.; Iizuka, M.; Masamune, O.; Kubo, N. A successful case of intraarterial prednisolone injection therapy in severe ulcerative colitis unresponsive to an intensive intravenous regimen. Gastroenterol. Jpn. 1993, 28, 725-729. [CrossRef] [PubMed]

10. Ito, A.; Iizuka, B.; Omori, T.; Nakamura, S.; Tokushige, K. Tacrolimus for remission induction and maintenance therapy in patients with ulcerative colitis: A retrospective evaluation study. Gastroenterol. Res. Pract. 2016, 2016, 5956316. [CrossRef] [PubMed]

11. Damião, A.O.M.C.; Azevedo, M.F.C.D.; Carlos, A.D.S.; Wada, M.Y.; Silva, T.V.M.; Feitosa, F.D.C. Conventional therapy for moderate to severe inflammatory bowel disease: A systematic literature review. World J. Gastroenterol. 2019, 25, 1142-1157. [CrossRef] [PubMed]

12. Löfberg, R.; Thomsen, O.O.; Langholz, E.; Schiöler, R.; Danielsson, A.; Suhr, O.; Graffner, H.; Påhlman, L.; Matzen, P.; Petersen, J.F.M. Budesonide versus prednisolone retention enemas in active distal ulcerative colitis. Aliment. Pharmacol. Ther. 1994, 8, 623-629. [CrossRef] [PubMed]

13. Lee, F.I.; Jewell, D.P.; Mani, V.; Keighley, M.R.; Kingston, R.D.; Record, C.O.; Grace, R.H.; Daniels, S.; Patterson, J.; Smith, K. A randomised trial comparing mesalazine and prednisolone foam enemas in patients with acute distal ulcerative colitis. Gut 1996, 38, 229-333. [CrossRef] [PubMed] 
14. Yano, H.; Hirayama, F.; Kamada, M.; Arima, H.; Uekama, K. Colon-specific delivery of prednisolone-appended alpha-cyclodextrin conjugate: Alleviation of systemic side effect after oral administration. J. Control. Release 2002, 79, 103-112. [CrossRef]

15. Wilson, J.C.; Sarsour, K.; Collinson, N.; Tuckwell, K.; Musselman, D.; Klearman, M.; Napalkov, P.; Jick, S.S.; Stone, J.H.; Meier, C.R. Serious adverse effects associated with glucocorticoid therapy in patients with giant cell arteritis (GCA): A nested case-control analysis. Semin. Arthritis Rheum. 2017, 46, 819-827.

16. Mundell, L.; Lindemann, R.; Douglas, J. Monitoring long-term oral corticosteroids. B.M.J. Open Qual. 2017, 6, e000209. [CrossRef] [PubMed]

17. Tozaki, H.; Fujita, T.; Odoriba, T.; Terabe, A.; Okabe, S.; Muranishi, S.; Yamamoto, A. Validation of a pharmacokinetic model of colon-specific drug delivery and the therapeutic effects of chitosan capsules containing 5-aminosalicylic acid on 2,4,6-trinitrobenzensulfonic acid-induced colitis in rats. J. Pharm. Pharmacol. 1999, 51, 1107-1112. [CrossRef]

18. Kim, H.J.; Neophytou, C. Natural anti-inflammatory compounds for the management and adjuvant therapy of inflammatory bowel disease and its drug delivery system. Arch. Pharm. Res. 2009, 32,997-1004. [CrossRef]

19. Naeem, M.; Kim, W.; Cao, J.; Jung, Y.; Yoo, J.-W. Enzyme/pH dual sensitive polymeric nanoparticles for targeted drug delivery to the inflamed colon. Colloids Surf. B. 2014, 123, 271-278. [CrossRef]

20. Takaya, T.; Ikeda, C.; Imagawa, N.; Niwa, K.; Takada, K. Development of a colon delivery capsule and the pharmacological activity of recombinant and human granulocyte colony-stimulating factor (rhG-CSF) in beagle dogs. J. Pharm. Pharmacol. 1995, 47, 474-478. [CrossRef]

21. Dianzani, C.; Cavalli, R.; Zara, G.P.; Gallicchio, M.; Lombardi, G.; Gasco, M.R.; Fantozzi, R. Cholesteryl butyrate solid lipid nanoparticles inhibit adhesion of human neutrophils to endothelial cells. Br. J. Pharmacol. 2006, 148, 648-656. [CrossRef] [PubMed]

22. Girlich, C.; Scholmerich, J. Topical delivery of steroids in inflammatory bowel disease. Curr. Drug Deliv. 2012, 9, 345-349. [CrossRef] [PubMed]

23. Lamprecht, A.; Ubrich, N.; Yamamoto, H.; Schäfer, U.; Takeuchi, H.; Maincent, P.; Kawashima, Y.; Lehr, C.M. Biodegradable nanoparticles for targeted drug delivery in treatment of inflammatory bowel disease. J. Pharmacol. Exp. Ther. 2001, 299, 775-781. [PubMed]

24. Xiao, B.; Xu, Z.; Viennois, E.; Zhang, Y.; Zhang, Z.; Zhang, M.; Han, M.K.; Kang, Y.; Merlin, D. Orally targeted delivery of tripeptide KPV via hyaluronic acid-functionalized nanoparticles efficiently alleviates ulcerative colitis. Mol. Ther. 2017, 25, 1628-1640. [CrossRef]

25. Collnot, E.M.; Ali, H.; Lehr, C.M. Nano and microparticulate drug carriers for targeting of the inflamed intestinal mucosa. J. Control. Release 2012, 161, 235-246. [CrossRef]

26. Tirosh, B.; Khatib, N.; Barenholz, Y.; Nissan, A.; Rubinstein, A. Transferrin as a luminal target for negatively charged liposomes in the inflamed colonic mucosa. Mol. Pharm. 2009, 6, 1083-1091. [CrossRef]

27. Hua, S.; Marks, E.; Schneider, J.J.; Keely, S. Advances in oral nano-delivery systems for colon targeted drug delivery in inflammatory bowel disease: Selective targeting to diseased versus healthy tissue. Nanomedicine 2015, 11, 1117-1132. [CrossRef]

28. Zhou, H.; Ichikawa, A.; Ikeuchi-Takahashi, Y.; Hattori, Y.; Onishi, H. Nanogels of succinylated glycol. chitosan-succinyl prednisolone conjugate: Preparation, in vitro characteristics and therapeutic potential. Pharmaceutics 2019, 11, 333. [CrossRef]

29. Kato, Y.; Onishi, H.; Machida, Y. Biological fate of highly-succinylated N-succinyl-chitosan and antitumor characteristics of its water-soluble conjugate with mitomycin $\mathrm{C}$ at i.v. and i.p. administration into tumor-bearing mice. Biol. Pharm. Bull. 2000, 23, 1497-1503. [CrossRef]

30. Crauste-Manciet, S.; Decroix, M.; Farinotti, R.; Chaumeil, J. Cefpodoxime-proxetil hydrolysis and food effects in the intestinal lumen before absorption: In vitro comparison of rabbit and human material. Int. J. Pharm. 1997, 157, 153-161. [CrossRef]

31. Hirayama, F.; Ogata, T.; Yano, H.; Arima, H.; Udo, K.; Takano, M.; Uekama, K. Release characteristics of a short-chain fatty acid, n-butyric acid, from its beta-cyclodextrin ester conjugate in rat biological media. J. Pharm. Sci. 2000, 89, 1486-1495. [CrossRef]

32. Udo, K.; Hokonohara, K.; Motoyama, K.; Arima, H.; Hirayama, F.; Uekama, K. 5-Fluorouracil acetic acid/beta-cyclodextrin conjugates: Drug release behavior in enzymatic and rat cecal media. Int. J. Pharm. 2010, 388, 95-100. [CrossRef] [PubMed] 
33. Onishi, H.; Ikeuchi-Takahashi, Y.; Kawano, K.; Hattori, Y. Preparation of chondroitin sulfate-glycyl-prednisolone conjugate nanogel and its efficacy in rats with ulcerative colitis. Biol. Pharm. Bull. 2019, 42, 1155-1163. [CrossRef] [PubMed]

34. Zhang, Y.; Casabianca, L.B. Probing Amino Acid Interaction with a Polystyrene Nanoparticle Surface Using Saturation-Transfer Difference (STD)-NMR. J. Phys. Chem. Lett. 2018, 9, 6921-6925. [CrossRef] [PubMed]

35. Oosegi, T.; Onishi, H.; Machida, Y. Gastrointestinal distribution and absorption behavior of Eudragit-coated chitosan-prednisolone conjugate microspheres in rats with TNBS-induced colitis. Int. J. Pharm. 2008, 348, 80-88. [CrossRef]

36. Onishi, H.; Kikuchi, H. Comparison of simple Eudragit microparticles loaded with prednisolone and Eudragit-coated chitosan-succinyl-prednisolone conjugate microparticles: Part II. In vivo evaluation of efficacy, toxicity, and biodisposition characteristics. Int. J. Mol. Sci. 2015, 16, 26125-26136. [CrossRef]

37. Kumria, R.; Nair, A.B.; Goomber, G.; Gupta, S. Buccal films of prednisolone with enhanced bioavailability. Drug Deliv. 2016, 23, 471-478. [CrossRef]

38. Onishi, H.; Oosegi, T.; Machida, Y. Efficacy and toxicity of Eudragit-coated chitosan-succinyl-prednisolone conjugate microspheres using rats with 2, 4, 6-trinitrobenzenesulfonic acid-induced colitis. Int. J. Pharm. 2008, 358, 296-302. [CrossRef]

39. Morris, G.P.; Beck, P.L.; Herridge, M.S.; Depew, W.T.; Szewczuk, M.R.; Wallace, J.L. Hapten-induced model of chronic inflammation and ulceration in the rat colon. Gastroenterology 1989, 96, 795-803. [CrossRef]

(C) 2020 by the authors. Licensee MDPI, Basel, Switzerland. This article is an open access article distributed under the terms and conditions of the Creative Commons Attribution (CC BY) license (http://creativecommons.org/licenses/by/4.0/). 